\title{
BMJ Open Renal replacement treatment initiation with twice-weekly versus thrice-weekly haemodialysis in patients with incident dialysis-dependent kidney disease: rationale and design of the TWOPLUS pilot clinical trial
}

\author{
Mariana Murea (D) , ${ }^{1}$ Shahriar Moossavi, ${ }^{1}$ Alison J Fletcher, ${ }^{1}$ Deanna N Jones, \\ Hiba I Sheikh, ${ }^{1}$ Gregory Russell, ${ }^{2}$ Kamyar Kalantar-Zadeh ${ }^{3}$
}

To cite: Murea M, Moossavi S, Fletcher AJ, et al. Renal replacement treatment initiation with twice-weekly versus thrice-weekly haemodialysis in patients with incident dialysis-dependent kidney disease: rationale and design of the TWOPLUS pilot clinical trial. BMJ Open 2021;11:e047596. doi:10.1136/ bmjopen-2020-047596

- Prepublication history and additional supplemental material for this paper are available online. To view these files, please visit the journal online (http://dx.doi.org/10.1136/ bmjopen-2020-047596).

Received 04 December 2020 Accepted 23 April 2021

Check for updates

(C) Author(s) (or their employer(s)) 2021. Re-use permitted under CC BY-NC. No commercial re-use. See rights and permissions. Published by BMJ.

For numbered affiliations see end of article.

Correspondence to

Dr Mariana Murea;

mmurea@wakehealth.edu

\section{ABSTRACT}

Introduction The optimal haemodialysis (HD)

prescription - frequency and dose-for patients with incident dialysis-dependent kidney disease (DDKD) and substantial residual kidney function (RKF)—-that is, renal urea clearance $\geq 2 \mathrm{~mL} / \mathrm{min} / 1.73 \mathrm{~m}^{2}$ and urine volume $\geq 500 \mathrm{~mL} /$ day - is not known. The aim of the present study is to test the feasibility and safety of a simple, reliable prescription of incremental $\mathrm{HD}$ in patients with incident DDKD and RKF.

Methods and analysis This parallel-group, openlabel randomised pilot trial will enrol 50 patients from 14 outpatient dialysis units. Participants will be randomised (1:1) to receive twice-weekly HD with adjuvant pharmacological therapy for 6 weeks followed by thrice-weekly HD (incremental HD group) or outright thrice-weekly HD (standard HD group). Age $\geq 18$ years, chronic kidney disease progressing to DDKD and urine output $\geq 500 \mathrm{~mL} /$ day are key inclusion criteria; patients with left ventricular ejection fraction $<30 \%$ and acute kidney injury requiring dialysis will be excluded. Adjuvant pharmacological therapy (ie, effective diuretic regimen, patiromer and sodium bicarbonate) will complement twice-weekly HD. The primary feasibility end points are recruitment rate, adherence to the assigned HD regimen, adherence to serial timed urine collections and treatment contamination. Incidence rate of clinically significant volume overload and metabolic imbalances in the first 3 months after randomisation will be used to assess intervention safety.

Ethics and dissemination The study has been reviewed and approved by the Institutional Review Board of Wake Forest School of Medicine in North Carolina, USA. Patient recruitment began on 14 June 2019, was paused between 13 March 2020 and 31 May 2020 due to COVID-19 pandemic, resumed on 01 June 2020 and will last until the required sample size has been attained. Participants will be followed in usual care fashion for a minimum of 6 months from last individual enrolled. All regulations and measures of ethics and confidentiality are handled in accordance with the Declaration of Helsinki.
Strengths and limitations of this study

- The pilot randomised controlled trial protocol employs a pragmatic approach to incremental-start haemodialysis (HD) based on broad eligibility criteria, practical dialysis management and in-centre clinical follow-up; intervention is tested in 14 dialysis units associated with one health system organisation.

- The study intervention consists of a structured schedule of incremental HD (time-delineated twiceweekly HD followed by thrice-weekly HD) to provide a cohesive and consistent approach for outcome assessment; however, the intervention of incrementalstart HD in this pilot study is not tailored beyond the first 6 weeks of the study and thus differs from other studies on incremental HD.

- Adjuvant pharmacological therapy (ie, loop diuretics, patiromer as potassium-binding agent and sodium bicarbonate) is combined with less frequent $\mathrm{HD}$.

- Blinding is not possible in respect to participants and intervention administrators.

- The study will inform key design questions required to deliver a successful intervention of incremental $H D$ in a larger, multicentre clinical trial.

Trial registration number NCT03740048; Pre-results.

\section{INTRODUCTION}

Almost all Americans with dialysis-dependent kidney disease (DDKD) are prescribed a standard haemodialysis (HD) regimen of fixed frequency (thrice-weekly) and fixed dose (dialysis single-pool Kt/V urea (spKt/Vurea) $\geq 1.2$, corresponding to standard Kt/Vurea (stdKt/Vurea) $\geq 2.1$ ). ${ }^{1}$ Whether this HD treatment is medically necessary for patients with new onset DDKD (ie, patients with incident DDKD) and ongoing, endogenous residual 
kidney function (RKF) as it is for those with long-term DDKD (ie, patients with prevalent DDKD) and no RKF is not known. The HD frequency of thrice-weekly was established as 'best-practice' following a long history of observed interventions and technological advancements in dialysis. ${ }^{23}$ The HD dose has been validated in clinical trials which involved solely patients with prevalent DDKD and no endogenous $\mathrm{RKF}^{45}$ Consequently, the optimal HD regimen (frequency and dose) for patients with incident DDKD and ongoing RKF - that is, renal urea clearance $\geq 2 \mathrm{~mL} / \mathrm{min} / 1.73 \mathrm{~m}^{2}$ and urine volume $\geq 500 \mathrm{~mL} /$ day-is not known as it has not been studied in prospective clinical trials.

Patients diagnosed with DDKD have various expressions of kidney disease, ranging from absence of urine output to more than $2 \mathrm{~L}$ of urine output in a 24-hour period. This ongoing kidney function, dubbed RKF, when present in the early stages of DDKD and if pharmacologically enhanced with adjuvant medical therapy, could confer safe and effective introduction of an initial dialysis regimen of less intensive HD. ${ }^{6-8}$ Based on mathematically-modelled urea kinetics, RKF levels with renal urea clearance $\geq 2 \mathrm{~mL} / \mathrm{min} / 1.73 \mathrm{~m}^{2}$ can accommodate prescriptions of less frequent $\mathrm{HD}$ in the form of once-weekly or twice-weekly HD. ${ }^{9}$ In these settings, RKF complements a dialysis dose of spKt/Vurea $\geq 1.2$ to attain total (dialysis + kidney) stdKt/Vurea of $\geq 2.1{ }^{10}$ Less frequent HD schedules can be maintained as long as clinical manifestations of severe kidney failure (eg, volume status, ultrafiltration rate with dialysis, nutrition) are adequately controlled and RKF is above the stated levels. ${ }^{910}$ With DDKD progression and as the level of RKF becomes insignificant, the prescription of HD can be amplified to 'standard' thrice-weekly HD regimens which later can be augmented to more intensive dialysis regimens. ${ }^{11} 12$ This gradual intensification of HD prescription including HD frequency, termed incremental HD, can be realised by changing either the HD frequency or the HD duration per session. ${ }^{12-16}$

Retrospective and observational data suggested that, compared with standard thrice-weekly HD, no worse and possibly better outcomes of RKF preservation, ${ }^{1718}$ patientreported quality of life ${ }^{1719}$ and possibly patient survival especially in the first months after dialysis transition ${ }^{20} \mathrm{can}$ be attained with less frequent schedules of HD in patients with incident DDKD and considerable RKF. Confounding these results is selection bias and lack of description of longitudinal changes in RKF with incremental schedules of HD relative to standard HD schedules. Other challenges in the application of incremental HD are apprehension about occult loss of RKF, with its attendant underdialysis and gradual development of volume overload, and patient non-compliance with time-consuming RKF assessments and changes in HD schedules. ${ }^{12} 21$ Herein we describe the protocol of a randomised pilot study that will investigate the feasibility of randomising 50 adults with incident DDKD and RKF to a regimen of incremental HD or standard HD.

\section{Box 1 Pilot study objectives}

Primary objective: Assess the feasibility and clinical safety of an intervention of incremental haemodialysis (HD) in patients with incident dialysis-dependent kidney disease and residual kidney function.

Feasibility will be assessed at 6 months as:

1. Recruitment rate.

2. Adherence to the assigned HD regimen.

3. Adherence to follow-up assessments of timed urine collection.

4. Control group cross-over (ie, receipt of twice-weekly HD in the control group).

Safety will be assessed in the first 3 months of the study based on the requirement of one or more additional HD treatment(s) or hospitalisation(s) for:

1. Volume overload.

2. Severe hyperkalaemia,

3. Severe metabolic acidosis.

4. Symptomatic uraemia.

Secondary objectives: Additional clinical outcomes will be compared at week 6 , month 3 , month 6 and month 12 after randomisation:

1. Changes in RKF.

2. Quality of life and psychological well-being (depression and anxiety).

3. Hospitalisation rate, management of volume status and electrolyte and acid-base homoeostasis.

4. Vascular access outcomes.

5. Anaemia, iron homoeostasis, bone-mineral metabolism management and nutritional state.

\section{METHODS AND ANALYSIS}

\section{Study objectives}

The overarching objective of the TWOPLUS trial is to answer key feasibility and design questions required to deliver a successful definitive trial of incremental HD. Box 1 summarises the primary and secondary objectives followed in this pilot clinical trial.

\section{Hypothesis}

Our main hypothesis is that incremental HD, when prescribed to patients with suitable RKF and planned as time-delineated regimen of twice-weekly HD with adjuvant pharmacological therapy followed by thrice-weekly $\mathrm{HD}$, will be feasible (ie, $\geq 2$ out of 4 feasibility metrics will be met) and will not compromise patient clinical safety (ie, rates of severe adverse events will be similar between the two treatment groups).

\section{Trial design}

This pilot study is parallel-group, open label, randomised controlled trial that combines standardised and personalised care. For the intervention of incremental HD, we adopted a uniform dialysis prescription in order to prevent potential errors and adverse events introduced with fluctuating levels of RKF and varying HD prescriptions. A total of 50 patients who fulfil all eligibility criteria and provide informed consent will be randomised, in 1:1 ratio, to receive one of the two HD regimens: (1) twiceweekly HD and clinically-indicated adjunctive pharmacological therapy (loop diuretic, potassium-binding agent (patiromer) and sodium bicarbonate) for six consecutive 


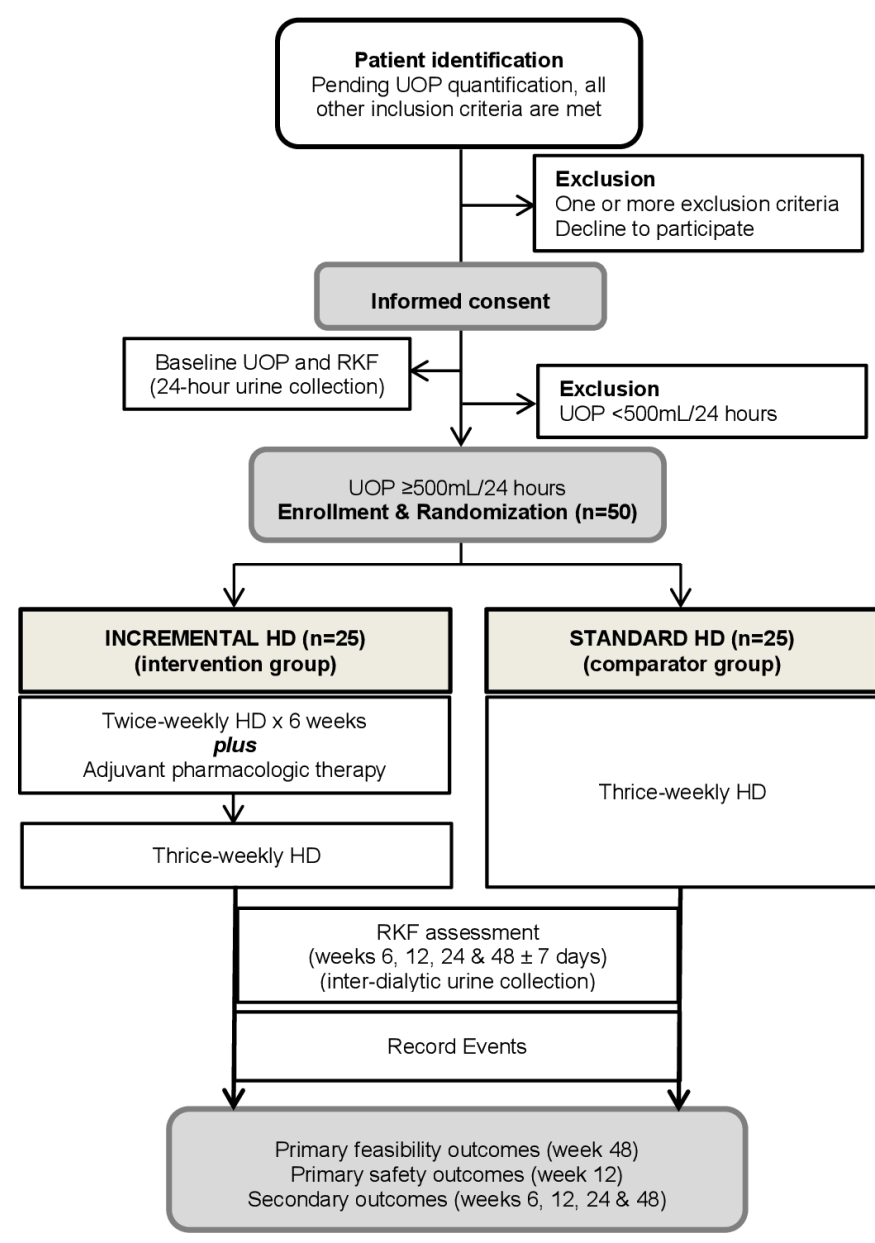

Figure 1 Study flow diagram.HD, haemodialysis; RKF, residual kidney function; UOP, urine output.

weeks, continued by thrice-weekly HD (incremental HD group); or (2) thrice-weekly HD (standard HD group) (figure 1).

\section{Setting and study population}

The study will be conducted at 14 outpatient dialysis units affiliated with a large academic tertiary centre in North Carolina, USA. Thirteen nephrologists currently treat over $600 \mathrm{HD}$ patients at these units. Adult patients with a diagnosis of DDKD initiated on treatment with chronic HD will be screened for eligibility criteria.

\section{Eligibility criteria and recruitment}

Eligibility criteria are listed in box 2. These were selected to defend against insufficient solute clearance and volume removal during the period of less frequent HD.

\section{Recruitment, randomisation and blinding}

Initial screening will take place with chart reviews of all patients with diagnosis of DDKD who are started on HD to identify those who initially qualify. Patients will be consented before any study-specific diagnostic test or randomisation (figure 1). The number of total incident patients with DDKD as well as the number of qualified and non-qualified subjects will be collected. Randomisation will be performed using a permuted block assignment

\section{Box 2 Eligibility criteria}

Inclusion criteria

- Age $\geq 18$ years.

- Require long-term maintenance HD as deemed by their treating nephrologist.

- Had eGFR $\geq 5 \mathrm{~mL} / \mathrm{min} / 1.73 \mathrm{~m}^{2}$ at the time of HD initiation.

- Received $\leq 6 \mathrm{HD}$ sessions by the expected date of enrolment and randomisation.

- Have urine output $\geq 500 \mathrm{~mL}$ per 24 -hour timed urine collection at the time of screening.

\section{Exclusion criteria}

- Abrupt decline in kidney function defined by eGFR $\geq 30 \mathrm{~mL} / \mathrm{min} / 1.73$ $\mathrm{m}^{2}$ within 3 months prior to HD initiation.

- Have severe systolic cardiac dysfunction with left ventricular ejection fraction $<30 \%$.

- Active diagnosis of hepatorenal syndrome.

- Have a malignancy that is likely to impact survival.

- Have a medical condition that would jeopardise the safety of the subject.

- History of non-compliance that would jeopardise patient adherence to study protocol.

- Inadequate written and verbal English comprehension.

eGFR, estimated glomerular filtration rate assessed by the Chronic Kidney Disease Epidemiology Collaboration equation; HD, haemodialysis.

scheme to ensure equal sized groups (intervention vs usual care). Allocation to incremental or standard HD will be stratified by type of primary vascular access used for $\mathrm{HD}$ at the time of enrolment (central venous catheter vs arteriovenous access).

Due to the nature of the intervention (ie, difference in frequency of HD treatment), the trial intervention will be unblinded. Bias and contamination could be introduced by a participant's inclination to assume less frequent HD treatments when randomised to control group or when expected to switch to more frequent HD. To minimise the effect of these potential problems, all participants will be educated about impending risks of less frequent HD when performed for an unchecked period of time. The patient information consent form has been written in an objective, neutral manner to accurately reflect current evidence, and does not discuss the hypothetical benefits of less frequent HD.

\section{Dialysis treatment allocation}

After randomisation, the HD prescription (frequency and time) and the pharmacological prescription (diuretics, potassium binders and acid buffers) will be determined based on treatment allocation. Patients assigned to the intervention group will receive a stepwise regimen of incremental HD whereby HD frequency-twice-weekly for 6 weeks followed by thrice-weekly HD-, HD time and prescription of diuretics, potassium binders and acid buffers is protocol-based. Under the direction of the treating nephrologist, progression from twice-weekly to thrice-weekly HD may occur prior to the 6 -week time point for clinical events that could benefit from increased 
Table 1 Elements of haemodialysis (HD) prescription and pharmacological therapy according to treatment allocation

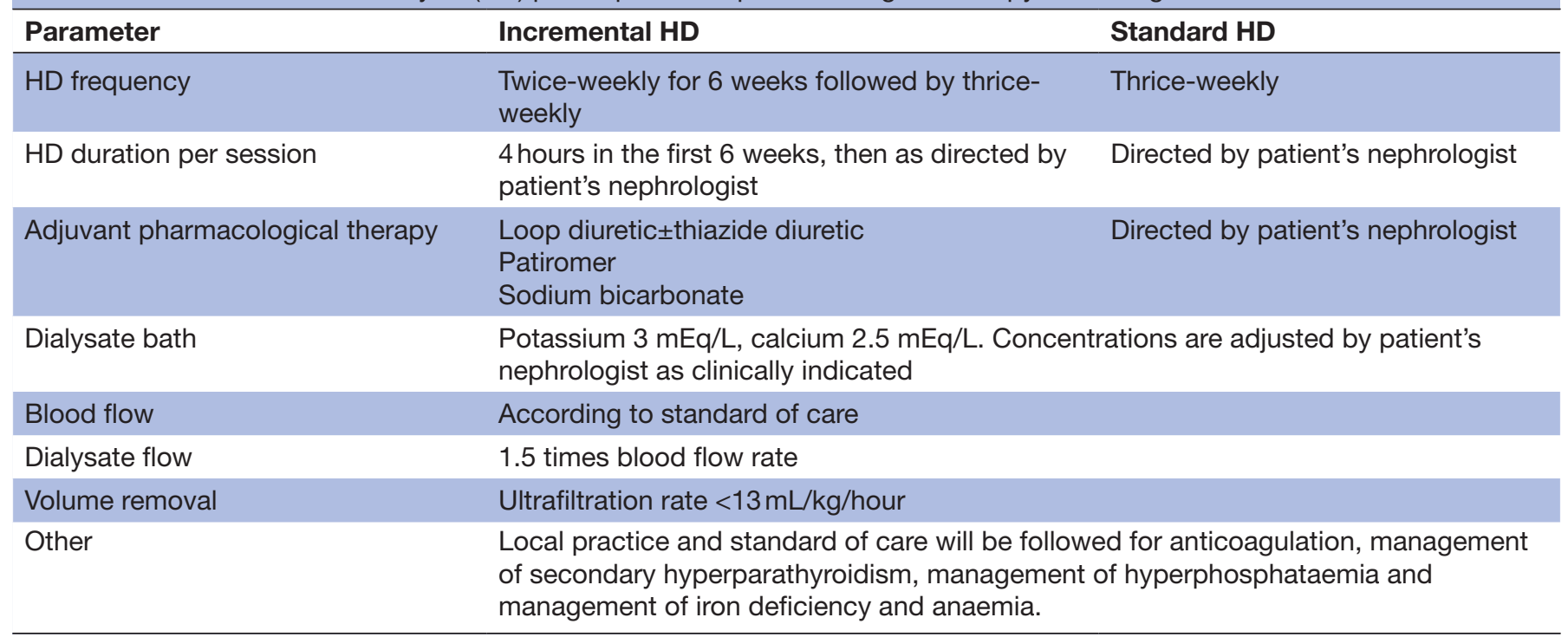

HD frequency, ultrafiltration rate $\geq 13 \mathrm{~mL} / \mathrm{kg} /$ hour per dialysis session and electrolyte or acid-based disorders that persist in spite of appropriate pharmacological and/ or dietary interventions. Patients assigned to the control group will be treated with a dialysis and pharmacological regimen set entirely by the treating nephrologist. The parameters of HD prescription, by group allocation, are summarised in table 1 . For practicality and in the absence of data regarding optimal level of urea solute clearance in patients with incident DDKD and RKF, the dialysis prescription will be adjusted to achieve dialysis singlepool Kt/Vurea $\left(\operatorname{spK}_{\mathrm{d}} \mathrm{t} /\right.$ Vurea) of $\geq 1.2$ and urea reduction ration (URR) of $\geq 65 \%$ in both treatment groups throughout the study period-including during the first 6 weeks in the incremental HD group.

\section{Adjuvant pharmacologic treatment}

Adjuvant pharmacological therapy (ie, loop diuretics \pm thiazide diuretics, potassium-binding agent and/or bicarbonate-based agent) will be prescribed in a study protocol-based manner during the period of twice-weekly HD in the incremental HD group. Diuretics will be used as follows: for patients prescribed loop diuretics prior HD initiation, the diuretic dose will be doubled and administered on non-HD days; for those not prescribed loop diuretics prior to HD initiation, furosemide at a dose of $80 \mathrm{mg}$ two times a day (or dose-equivalent torsemide or bumetanide) will be prescribed on non-HD days. Based on clinical indications, the dose of furosemide will be titrated to a maximum dose of $320 \mathrm{mg} /$ day. Based on its proven efficacy and safety in clinical trials involving patients with chronic kidney disease and hyperkalaemia, patiromer will be prescribed as the potassium-binding agent of choice. ${ }^{22-24}$ Treatment with patiromer will be instituted for serum potassium levels of $\geq 5.1 \mathrm{mEq} / \mathrm{L}$. The cut-off level for serum potassium and patiromer administration was elected on the basis of uncertain patient adherence to the prescribed dialysis regimen, prescription of potassium dialysate bath of $3 \mathrm{mEq} / \mathrm{L}$ in all patients at the time of study enrolment and continuation of renin-angiotensin-aldosterone system inhibitors in patients randomised to twice-weekly HD. Patiromer will be administered on non-HD days and the dose will be titrated to maintain pre-HD serum potassium levels between $4.6-5.3 \mathrm{mEq} / \mathrm{L}{ }^{25}$ Sodium bicarbonate will be prescribed for treatment and/or prevention of metabolic acidosis, aiming for target pre-HD serum bicarbonate level of 20-22 mEq/L. ${ }^{26} 27$ Recommendations to changes in adjuvant pharmacological treatment under the guidance of the treating nephrologist will be permitted. After the period of twice-weekly HD and once the patients convert to thrice-weekly HD, patiromer will be discontinued; and diuretic prescription will be directed by the treating nephrologist. Medications from the category of renin-angiotensin-aldosterone system inhibitors will not be discontinued given the protocol includes purposeful use of loop diuretics and patiromer. All medications prescribed to participants randomised to standard HD will be guided by the treating nephrologist throughout the study.

\section{Measurements and schedule of assessments}

Table 2 summarises data collection and the schedule of assessments. Study-specific assessments will take place at enrolment and at weeks $6,12,24$ and 48 ( \pm 7 days). Timed urine collection at baseline will consist of 24-hour collection (done on a non-dialysis day); at follow-up will consist of inter-dialytic collection, starting at the end of the first HD session and ending at the beginning of the second HD session during the week of assessment. Measurements performed on timed urine collections will include volume, urea, creatinine and beta- 2 microglobulin concentration.

\section{Evaluation of solute clearance}

Clearance of uraemic solute retention will be evaluated for small molecular weight substances (ie, urea) and 
Table 2 Data collection and schedule of assessments

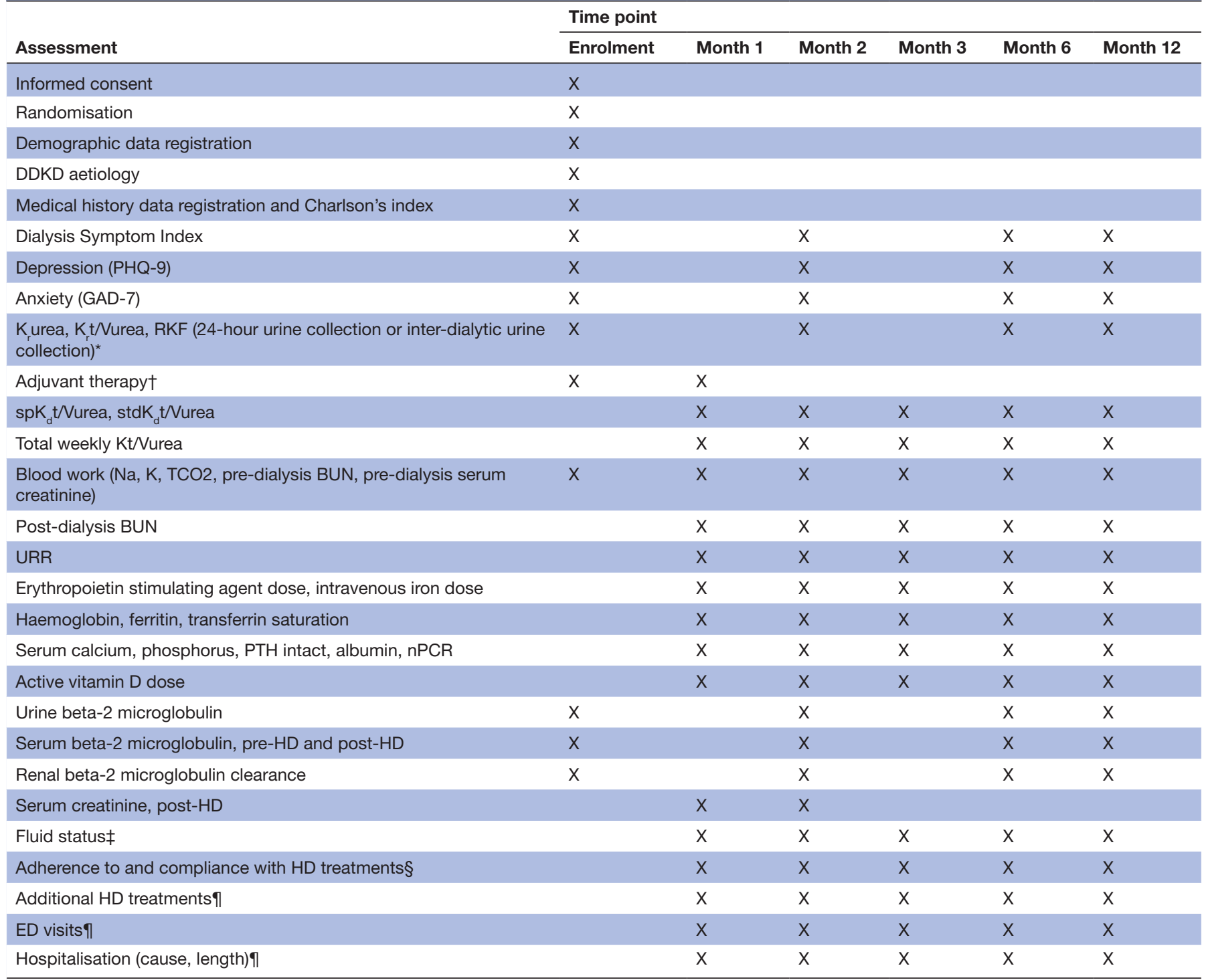

Baseline laboratory studies will be considered as the most recent available values within 2 weeks prior to HD initiation.

stdK $_{d} t /$ Vurea month 1, based on average $\operatorname{spK}_{d} t /$ Vurea month 1 (weeks 2 and 4); stdK $_{d} t /$ Vurea month 2, based on average spK $t /$ Vurea month 2 (weeks 6 and 8); stdK $t$ /Vurea month 3, based on average spK t/Vurea month 3 (weeks 9 and 12).

Total Kt/Vurea month 1, based on stdK $\mathrm{t} /$ Vurea month 1 and baseline $\mathrm{K} t /$ Vurea; total Kt/Vurea month 2, based on stdK $\mathrm{t} /$ Vurea month 2 and $\mathrm{K} t /$ Vurea obtained during week 6; total Kt/Vurea month 3 , based on stdK $\mathrm{d} t /$ Vurea month 3 and $\mathrm{K}_{\mathrm{r}} \mathrm{t} /$ Vurea obtained during week 12 ; total Kt/Vurea month 6, based on stdK $\mathrm{d}$ //Vurea month 6 and $\mathrm{K} t$ /Vurea obtained during week 24; total Kt/Vurea month 12, based on stdK $\mathrm{t} /$ /Vurea month 12 and $\mathrm{K}_{\mathrm{t}} \mathrm{t} /$ Vurea obtained during week 48.

*24-hour urine collection will be obtained at baseline (within 2 weeks before or after first HD treatment). This will be used to calculate baseline $\mathrm{K} \mathrm{t} /$ Vurea and baseline renal beta-2 microglobulin clearance. Inter-dialytic urine collections will be done at week $6,12,24$ and $48 \pm 7$ days. Measurements performed on urine collections will include: urine volume, urine urea, urine creatinine and urine beta-2 microglobulin concentration. With inter-dialytic urine collection, blood work (BUN, serum creatinine and serum beta-2 microglobulin levels) will be done post-HD and pre-HD corresponding to the HD treatments at the beginning and at the end, respectively, of the inter-dialytic collection.

†Adjuvant therapy will include diuretics (loop diuretic \pm thiazide), patiromer and sodium bicarbonate.

†Fluid status assessment based on inter-dialytic weight gain (expressed as percentage(\%) of estimated dry weight), residual weight (post-dialysis weight-estimated dry weight; expressed as \% of estimated dry weight) and ultrafiltration rate (calculated as $\mathrm{mL} / \mathrm{kg} / \mathrm{hour}$ per dialysis session; goal $<13 \mathrm{~mL} / \mathrm{kg} / \mathrm{hour}$ ) §Proportion of missed HD treatments and proportion of shortened HD treatments.

IRequirement of additional HD treatment(s) and/or hospitalisation(s) for fluid or metabolic imbalances.

BUN, blood urea nitrogen; DDKD, dialysis-dependent kidney disease; ED, emergency department; GAD-7, Generalized Anxiety Disorder-7; HD, haemodialysis; KDQOL-SF36, kidney disease quality of life short form 36; $\mathrm{K} t$ /Vurea, residual renal urea clearance; $\mathrm{nPCR}$, normalised catabolic protein rate; $\mathrm{PHQ}-9$, Patient Health Questionnaire-9; RKF, residual kidney function; $\mathrm{spK}_{\mathrm{d}} \mathrm{t} /$ Vurea, single-pool dialysis urea clearance; stdK $\mathrm{t} /$ Vurea, standard dialysis urea clearance; $\mathrm{TCO}$, total carbon dioxide; URR, urea reduction ratio.

middle molecular weight molecules (ie, beta-2 microglobulin). ${ }^{28-30}$ Adequacy of urea clearance will be assessed with the following metrics: (1) $\operatorname{spK}_{\mathrm{d}} \mathrm{t}$ /Vurea, based on blood urea nitrogen levels collected before and immediately after HD treatment, calculated as dialysis clearance of urea $(\mathrm{K}, \mathrm{mL} / \mathrm{min})$ multiplied by time ( $\mathrm{t}, \mathrm{min})$ and normalised to the volume of distribution of urea $(\mathrm{V}, \mathrm{mL})$ based on Watson formula, (2) URR, based on pre-HD and post-HD blood urea nitrogen levels and (3) standard weekly dialysis urea clearance $\left(\operatorname{stdK}_{\mathrm{d}} \mathrm{t} /\right.$ Vurea) and 
total Kt/Vurea. ${ }^{131}$ Weekly residual renal urea clearance $\left(\mathrm{K}_{\mathrm{r}} \mathrm{t} /\right.$ Vurea) will be calculated from residual renal urea clearance $(\mathrm{mL} / \mathrm{min})$ and using time-averaged blood urea nitrogen concentration during the collection period. ${ }^{32}$ $\operatorname{RKF}\left(\mathrm{ml} / \mathrm{min} / 1.73 \mathrm{~m}^{2}\right)$ will be calculated as arithmetic mean of urea and creatinine clearance, with body surface area correction. To compare total urea clearance delivered over a week period between the two treatment groups, we will calculate total Kt/Vurea as the sum of weekly dialysis urea clearance $\left(\operatorname{stdK}_{\mathrm{d}} \mathrm{t} /\right.$ Vurea, calculated based on $\operatorname{spK}_{\mathrm{d}} \mathrm{t} /$ Vurea) and weekly residual renal urea clearance $\left(\mathrm{K}_{\mathrm{r}} \mathrm{t} /\right.$ Vurea) using the kinetic model of urea with the formula modified from Gotch. ${ }^{33}$ Renal clearance and mass removal for beta-2 microglobulin (marker of middle molecule clearance) will be calculated on timed urine collection, urine solute and blood solute concentration. ${ }^{34}$ Formulas and equations that will be used to evaluate solute clearance are listed in online supplemental table 1 .

\section{Recruitment period}

Patient recruitment began on 14 June 2019, was paused between 13 March 2020 and 31 May 2020 due to COVID-19 pandemic and resumed on 01 June 2020. Recruitment will be continued until the required sample size has been achieved.

\section{Outcome measurements}

Follow-up will commence at the time of enrolment and will continue for a minimum of 6 months. The follow-up time (days) will be the difference in days from the date of the end of the follow-up minus the date of the baseline visit. Patients will be censored if alive at the end of study follow-up, receive a renal transplant, recover renal function, transfer to another facility, transition to different dialysis modality (eg, peritoneal dialysis, home HD), are lost from follow-up or withdraw their informed consent.

\section{Primary feasibility outcomes}

This pilot study will be considered successful based on attaining the following feasibility outcomes assessed as: (1) $\geq 70 \%$ of eligible patients are recruited, (2) $\geq 95 \%$ of participants randomised in the intervention group will adhere to the HD regimen, (3) $\geq 80 \%$ patients adhere to study-specific timed urine collection and $(4) \leq 5 \%$ of participants randomised in the control group will cross over to a regimen of less frequent HD. Feasibility metrics were selected based on a consensus opinion among investigators regarding medically acceptable rates of adherence to the tested intervention.

\section{Primary safety outcomes}

The intervention of stepwise HD schedule will be considered safe, relative to standard HD schedule, if there will be no significant difference in severe adverse events between the two HD treatment groups during the first 3 months of the study.

Severe adverse event(s) will be defined as the requirement of one or more additional HD treatment(s) or hospitalisation(s) for: (1) a condition related to poor volume control (ie, clinically-determined volume overload, decompensated heart failure or hypertensive urgency/emergency); (2) severe electrolyte imbalance (ie, serum potassium $>6.5 \mathrm{mEq} / \mathrm{L}$ ); (3) severe metabolic acidosis (ie, serum bicarbonate $<15 \mathrm{mEq} / \mathrm{L}$ ); (4) symptomatic uraemia (ie, uraemic encephalopathy, uraemic pericarditis); or (5) death of any of the participants associated with the pilot study.

\section{Secondary outcomes}

Secondary outcomes will encompass clinical $^{35}$ and patient-reported outcomes. ${ }^{36-40}$ These will be compared between the two treatment groups at weeks $6,12,24$ and 48 ( \pm 7 days) after randomisation (online supplemental table 2). Patients with significant cognitive impairment (determined by total score $<3$ on Mini-Cog testing) or active psychosis will be excluded from questionnaire testing. ${ }^{41}$

\section{Data collection}

Demographic and clinical variables will be collected at study enrolment: age, sex, race, height, body surface area, body mass index, DDKD aetiology, HD initiation date, comorbidities at HD initiation, nephrology care prior to dialysis initiation (first outpatient nephrology visit and number of outpatient nephrology office visits) prior to dialysis initiation, vascular access(es) present and/or used for HD at dialysis initiation, date and type of arteriovenous access placed (as applicable), body weight before dialysis initiation, estimated dry weight at dialysis initiation and comorbid conditions present at dialysis initiation. All clinical events, vascular access complications, vascular access interventions and hospitalisations following randomisation will be recorded. As part of the standard of care, dialysis $\mathrm{spK}_{\mathrm{d}} \mathrm{t} /$ Vurea, URR, complete metabolic panel, complete blood count, iron profile and mineral and bone profile are obtained every 2-4 weeks at the outpatient dialysis units. Total weekly Kt/Vurea on months 1, 2, 3, 6 and 12 will include the contributions of weekly $\mathrm{K}_{\mathrm{r}} \mathrm{t}$ /Vurea calculated from timed urine collections performed at baseline and weeks 6, 12, 24 and 48, respectively; and weekly $\operatorname{stdK}_{\mathrm{d}} \mathrm{t} /$ Vurea calculated based on $\operatorname{spK}_{\mathrm{d}} \mathrm{t} /$ Vurea $^{42}$ obtained on months 1, 2, 3, 6 and 12, respectively. All the laboratory data will be analysed at one single central laboratory (Meridian Laboratory Corporation, Charlotte, North Carolina, USA).

\section{Concomitant care}

Both treatment groups will continue to receive medical and pharmacological management of comorbid conditions as well as standard lifestyle advice according to prevailing guidelines. ${ }^{43}$ Dry weight estimation and adjustment will be at the decision of the treating nephrologist in both study groups.

\section{Sample size calculation}

Traditional sample size determination is made to ensure specific power to detect treatment effects. However, the 
primary purpose of this pilot study was to evaluate study feasibility. Moreover, no comparative clinical data exist on effects of incremental HD versus standard HD on longitudinal changes in RKF or other clinical outcomes in patients with incident DDKD. Therefore, pilot trial sample size of 50 patients total (25 patients per treatment group) is consistent with recommendations for pilot and feasibility studies where samples of 10-20 participants per group have been deemed adequate to assess feasibility outcomes. ${ }^{45}$

\section{Statistical analysis}

Feasibility will be assessed using descriptive data regarding eligible patients, enrolled patients, intervention compliance and adherence to study-specific assessments and drop-out/withdrawal rate among each intervention. The proportion of people meeting each of the feasibility and protocol adherence end points with accompanying 95\% CIs will be estimated using skew-corrected score tests with a continuity correction. Progress from pilot to large scale trial will be considered as: (1) continue the study without modifications (feasible as is) if all feasibility criteria are met; (2) continue with protocol modifications (feasible with modifications); or (3i) stop the main study (not feasible) if none of the four feasibility criteria was met.

For evaluation of primary safety end points and secondary clinical end points, intention-to-treat principles will be followed. All data will be tested for normality and equal variance. In the absence of normality or constant variance, the data will be transformed using natural log or appropriate transformation. To account for differences between populations and increase power, an analysis of covariance modelling approach will be considered to take into account possible covariate adjustments of baseline characteristics (ie, nephrology care prior to dialysis initiation, comorbidities). Evaluation of categorical end points will be performed using $\chi^{2}$ tests. Evaluation of continuous end points will be performed using Student's unpaired t-test for normally distributed variables, or the Wilcoxon rank-sum test for non-parametric data. For comparison of outcomes measured at all time points during the study, a repeated measures analysis of variance with two factors (time $\times$ group) will be used. The consistency of effects on safety end points and secondary clinical outcomes will be explored in a variety of subgroups (ie, based on age, vascular access used at HD enrolment, and baseline level of RKF). Kaplan-Meier methods will be used for time-to-first hospitalisation analyses. Missing RKF data for subjects will be replaced (rather than excluding them from the analysis, as missing RKF measurements might not be completely at random) using linear regression to estimate values based on other measurements for each subject (linear trend at point, SPSS). No adjustments for multiplicity will be made.

\section{Patient and public involvement}

Patients or the public were not involved in the design or conduct of the pilot trial. Feedback from participants in this pilot trial will be obtained to (1) evaluate the delivery of study-related information, (2) evaluate coordination of care during the study as it pertained to study-related timed urine collections, (3) evaluate patient perceptions on study-related assessments, (4) elicit patients' motivations for study participation and (5) obtain participant input regarding future research concerning incremental HD. A patient feedback questionnaire, structured into five domains (information and communication, coordination of care, perception on study-related assessments, motivation and future studies) each containing four to six items with answers rated on a 5-level scale, will be administered to study participants (online supplemental table 3 ). Feedback from funders will be obtained at the completion of the study to assess whether future funding for larger clinical trial is feasible. Furthermore, we will consult with dialysis staff members (eg, nurses, managers) and physicians to refine the programme of incremental HD to ensure smooth application across other health systems.

\section{ETHICS AND DISSEMINATION}

\section{Research ethics approval}

This study was reviewed and approved by the Wake Forest School of Medicine Institutional Review Board (IRB) (IRB00054726) on 19 February 2019, and the trial was registered on 14 November 2018 at ClinicalTrials. gov. Amendments to the protocol will be submitted to the Wake Forest School of Medicine IRB for review and approval. The study will be conducted in accordance with the principles stated in the Declaration of Helsinki. ${ }^{46}$

\section{Consent to participate}

Patients must provide informed consent, either written or verbal, before study-specific 24-hour urine collection is performed. If study personnel are unable to contact the patient to obtain written informed consent through in-person recruitment prior to obtaining 24-hour urine collection to evaluate RKF, they will contact the patient to obtain verbal informed consent via telephone. To obtain verbal consent, study personnel will use an IRB-approved telephone screening script to describe the study and an IRB-approved oral consent script to obtain verbal consent, and will then document the date on which verbal informed consent was obtained. The Wake Forest School of Medicine IRB approved the procedure for verbal consent. All patients must have a written informed consent before randomisation. Participants will be notified of any significant changes to the study design via a mailed letter using the information collected at the time of recruitment.

\section{Participant and data confidentiality}

Only the study personnel at Wake Forest Outpatient Dialysis Units and Wake Forest Baptist Medical Center will have access to identifying patient information. Wake Forest study team members will receive data about participating patients when there will be lack of adherence 
to treatment protocol (eg, refusal to switch from twiceweekly to thrice-weekly HD) but will have no direct patient contact with the participants. Study data will be collected and managed using electronic data capture tools hosted at Wake Forest Outpatient Dialysis Research and Administration Center. Data will be stored for 7 years according to Health Insurance Portability and Accountability Act (HIPAA) requirements. All study personnel have received requisite training in data confidentiality and human subjects research.

\section{Data safety and trial monitoring}

The IRB of the Wake Forest School of Medicine determined that a data monitoring committee was not necessary for this pilot clinical trial due to minimal participant risk. Data monitoring will be conducted and reported by the principal investigator (PI) as projected by the data safety monitoring plan. The PI will immediately report any unanticipated adverse events, all severe adverse events and all study deviations to the Wake Forest School of Medicine IRB.

\section{Dissemination plan}

We will submit the findings of this pilot randomised clinical trial for peer-reviewed publication. Authorship eligibility will be determined using International Committee of Medical Journal Editors (ICMJE) guidelines. ${ }^{47}$ Results will be presented at national and international conferences. Data resulted from this pilot randomised clinical trial will be available on reasonable request.

\section{Translatability}

In order to increase the likelihood of widespread adoption, interventions for incremental HD must be readily integrated into routine HD care and applicable to the majority of HD patients. This study uses limited exclusion criteria and the intervention described can be highly translatable to other HD units. In order to gauge the acceptability of our intervention, a flowchart of patients approached and those consented will be recorded. We will record reasons for study refusal from those that decline to participate. Additionally, a detailed log of completion and timeliness of timed urine collection will be kept in order to help translate the intervention into clinical practice. At the end of the study, investigators will meet to discuss the study findings and identify items that require action and refine the intervention of stepwise HD for a future multicentre clinical trial. Revisions to the study curriculum will be made in response to these data with the goal of making the intervention as simple and generalisable as possible to the largest number of HD patients.

\section{Strengths and limitations}

The proposed pilot trial has a number of strengths. The study will employ a pragmatic approach to assess the benefit and/or harm, patient quality of life and compliance with a pre-defined, incremental schedule of HD which combines standardised care with personalised treatments. Incremental HD regimen will be delivered at inpatient and outpatient HD units and the intervention was designed to be translatable to other HD units. The protocol was developed using the Standard Protocol Items: Recommendations for Interventional Trials guidelines for a pilot randomised clinical trial protocol, increasing the likelihood that important study design elements have been addressed. We acknowledge in a subsequent multicentre study the rate of recruitment may be different (lower) than the rate targeted in the pilot study. For any cross-sectional cohort of patients with incident DDKD, the recruitment rate will not only depend on inclusion/exclusion criteria but also vary by country/ state, region and local practices. Using the framework presented by Robert Newcombe, we calculated a $95 \%$ CI of $(56.3 \%, 80.9 \%)$ around the estimated $70 \%$ recruitment rate across different clinical centres. ${ }^{48}$

Our study also has limitations. First, non-Englishspeaking patients are excluded from the study, which limits its generalisability to non-English-speaking dialysisdependent populations. The findings from this study will help to justify developing materials in alternative languages that are accessible to a wider range of dialysis patients in future larger studies. Second, this pilot trial is conducted at one large health system organisation. However, we believe this study is an important first step in establishing the protocol for incremental HD across other organisations. Finally, the intervention of twice-weekly HD for a limited period of 6 weeks restricts the evaluation of long-term impact of less frequent HD on changes in RKF. Nevertheless, the primary objective of this pilot is to test patient adherence to changes in HD prescription and serial urine collections. To date, no prospective trials studied patients' compliance with incremental HD and associated assessments. Observational and retrospective studies harbour many potential confounders such as inclusion of healthier and compliant patients for whom the treating nephrologist considered incremental HD a safe therapy plan. ${ }^{12}$ Based on clinical experience, we deemed patients who fulfil the selected eligibility criteria will have a minimum 6-week period of adequate levels of RKF to allow safe prescription of twice-weekly HD.

\section{DISCUSSION}

The TWOPLUS trial will address key feasibility and safety issues facing future definitive trials of incremental HD in patients with incident DDKD. Feasibility will be appraised as institutional and patient-based practicality of incremental HD. The outcome measure for institutional-based feasibility will be patient eligibility and enrolment statistics. Outcome measures for patient-based feasibility will be patient adherence, compliance, contamination and withdrawal statistics. Patient safety will be assessed based on the incidence rate of clinically significant volume overload and metabolic imbalances. The results will be used to optimise study design and determine the number of centres needed to participate for a future multicentre clinical trial with a primary composite end point of 
all-cause death and patient-reported quality of life, enrolment period of 2 years and follow-up period of 3 years.

Several design aspects of this pilot study require justification. First, compared with a broader set of eligibility criteria, ${ }^{13}$ the inclusion criteria selected in this pilot were simplified given the protocol included prescription of diuretics, a potassium binding agent and sodium bicarbonate. The investigators considered cut-off levels for 24-hour urine output and baseline estimated glomerular filtration rate (eGFR) that would confer satisfactory levels of RKF and volume control. We believe these inclusion criteria are more readily applicable in real-life clinical practice. In addition, participant inclusion based on baseline eGFR $\geq 5 \mathrm{~mL} / \mathrm{min} / 1.73 \mathrm{~m}^{2}$, defined as the most recent eGFR prior to HD initiation, rather than urinebased clearance studies will streamline patient enrolment without jeopardising medical safety while urine-based clearance studies are being processed. Second, the type of vascular access used for HD does not disqualify study participation as long as the patient has a diagnosis of DDKD and is anticipated to require long-term maintenance HD. Third, the intervention of twice-weekly HD is limited to 6 weeks given the gap in knowledge regarding patients' acceptance to change to thrice-weekly HD and the goal to obtain information across all participants regarding their compliance with the recommended HD schedule. Finally, although most feasibility outcomes will be collected within 3 months of randomisation, participants will be followed for a minimum of 6 months and up to 12 months in order to gather longitudinal data on changes in RKF. The study will provide constructive information to optimise the approach to incremental HD in future studies as either (1) personalised HD, that is, twice-weekly HD at dialysis initiation, converted to thrice-weekly HD based on changes in RKF, should the pilot indicate satisfactory adherence; or (2) stepped HD, that is, twice-weekly HD at dialysis initiation, converted to thrice-weekly HD at a pre-defined time point, should the pilot indicate inadequate patient adherence.

Less frequent HD cannot be performed in all patients or for limitless duration. It requires attention to many details and is more labour intensive for the dialysis staff. A relevant concern with incremental HD is the risk of inadequate dialysis when patients on less frequent HD lose RKF and this goes unrecognised while the dialysis dose is not augmented. Additional concerns are that an incremental HD approach will require a labour-intensive process to gain more patient understanding and cooperation than the conventional HD schedule. In addition, the ideal method of quantifying RKF requires a timed urine collection followed by blood tests with appropriate computations to take into account the duration of collected urine and the timing of the last HD. ${ }^{32}{ }^{49}$ Under-collection or over-collection of urine over a 24-hour period is not uncommon in practice, and no methods can reliably assess the accuracy of 24-hour urine collection. ${ }^{50}$ Logistical challenges of obtaining, analysing and reporting of timed urine collections are some of the reasons why
RKF is not more often used in routine clinical care of HD patients. Difficulties in quantifying RKF through timed urine collections recognised, several investigators examined methods of estimating RKF that use commonly available laboratory and clinical data, without reliance on urine collection; yet, based on available research, these methods are less accurate than urine-based RKF measurements. ${ }^{51-53}$ Moreover, the relationship between the duration of twice-weekly HD and patient outcomes or adherence to dialysis regimen has not been studied, and a theoretical concern is that an unchecked, prolonged period of less frequent HD could be detrimental to the health of the patients with DDKD.

Attention to customising dialysis schedules in patients with DDKD is surging. ${ }^{94-56}$ While this is the first clinical trial of incremental HD in the USA, clinical trials on this theme have been ongoing in other parts of the world. ${ }^{10}{ }^{57-59}$ The framework of this pilot clinical trial offers an organised and integrated approach to dialysis treatments in patient with incident DDKD. The study design and our model of stepped HD schedule as a form of incremental HD is intended to be sufficiently structured to provide a cohesive and consistent approach for effective outcomes assessment while being sufficiently flexible for physicians to tailor dialysis treatments based on estimated level of kidney function at dialysis initiation. We elected a period of 6 weeks for the schedule of twiceweekly HD. With future studies and once the trajectory of RKF with alternate schedules of HD is better defined, the period of less frequent HD can be further customised based on patient characteristics.

In conclusion, the findings from this pilot randomised clinical trial will inform the feasibility and safety of a definitive trial powered to detect the effectiveness and establish the safety of incremental HD (ie, twice-weekly to thrice-weekly HD) in patients with incident DDKD.

\section{Trial status}

Patient recruitment began on 14 June 2019, was paused between 13 March 2020 and 31 May 2020 due to COVID-19 pandemic, resumed on 01 June 2020 and will last until the required sample size has been attained. Participants will be followed in usual care fashion for a minimum of 6 months from last individual enrolled. At the time of manuscript submission, $48 \%$ (24 participants) were recruited.

\section{Author affiliations}

${ }^{1}$ Department of Internal Medicine, Section on Nephrology, Wake Forest School of Medicine, Winston-Salem, North Carolina, USA

${ }^{2}$ Department of Biostatistics and Data Science, Wake Forest School of Medicine, Winston-Salem, North Carolina, USA

${ }^{3}$ Division of Nephrology, University of California Irvine School of Medicine, Irvine, California, USA

Contributors MM designed the study. KK-Z participated in study conception. SM, AJF, DNJ and HIS participated in study implementation. GR provided statistical expertise in clinical trial design. All authors contributed to refinement of the study protocol and approved the final manuscript. 
Funding The study is investigator initiated, sponsored by Relypsa, Vifor Pharma (IE19-00819/GTS47902).

Competing interests The principal investigator (MM) received funding for this trial from Relypsa, Vifor Pharma. KK-Z has received commercial honoraria and/ or support from Abbott, AbbVie, Alexion, AMAG Pharma, Amgen, AstraZeneca, Aveo, Baxter, Chugai, DaVita, Dr Schaer, Fresenius, Genentech, Haymarket Media, Hospira, Kabi, Keryx, National Institutes of Health, Novartis, PCORI, Pfizer, Relypsa, Resverlogix, Sandoz, Sanofi, Shire, UpToDate, Vifor and ZS Pharma.

Patient consent for publication Not required.

Provenance and peer review Not commissioned; externally peer reviewed.

Supplemental material This content has been supplied by the author(s). It has not been vetted by BMJ Publishing Group Limited (BMJ) and may not have been peer-reviewed. Any opinions or recommendations discussed are solely those of the author(s) and are not endorsed by BMJ. BMJ disclaims all liability and responsibility arising from any reliance placed on the content. Where the content includes any translated material, BMJ does not warrant the accuracy and reliability of the translations (including but not limited to local regulations, clinical guidelines, terminology, drug names and drug dosages), and is not responsible for any error and/or omissions arising from translation and adaptation or otherwise.

Open access This is an open access article distributed in accordance with the Creative Commons Attribution Non Commercial (CC BY-NC 4.0) license, which permits others to distribute, remix, adapt, build upon this work non-commercially, and license their derivative works on different terms, provided the original work is properly cited, appropriate credit is given, any changes made indicated, and the use is non-commercial. See: http://creativecommons.org/licenses/by-nc/4.0/.

ORCID iD

Mariana Murea http://orcid.org/0000-0003-3217-1691

\section{REFERENCES}

1 National Kidney Foundation. KDOQI clinical practice guideline for hemodialysis adequacy: 2015 update. Am J Kidney Dis 2015;66:884-930.

2 Gottschalk CW, Fellner SK. History of the science of dialysis. Am J Nephrol 1997;17:289-98.

3 Blagg CR. The early history of dialysis for chronic renal failure in the United States: a view from Seattle. Am J Kidney Dis 2007;49:482-96.

4 Lowrie EG, Laird NM, Parker TF, et al. Effect of the hemodialysis prescription of patient morbidity: report from the National cooperative dialysis study. N Engl J Med 1981;305:1176-81.

5 Eknoyan G, Beck GJ, Cheung AK, et al. Effect of dialysis dose and membrane flux in maintenance hemodialysis. $N$ Engl J Med 2002;347:2010-9.

6 Shafi T, Mullangi S, Toth-Manikowski SM, et al. Residual kidney function: implications in the era of personalized medicine. Semin Dial 2017;30:241-5.

7 Shafi T, Levey AS. Measurement and estimation of residual kidney function in patients on dialysis. Adv Chronic Kidney Dis 2018;25:93-104.

8 Murea M, Kalantar-Zadeh K. Incremental and twice-weekly hemodialysis program in practice. Clin J Am Soc Nephrol 2020;16:147-9.

9 Basile C, Casino FG, EUDIAL Working Group of ERA-EDTA. Incremental haemodialysis and residual kidney function: more and more observations but no trials. Nephrol Dial Transplant 2019;34:1806-11.

10 Casino FG, Basile C, Kirmizis D, et al. The reasons for a clinical trial on incremental haemodialysis. Nephrol Dial Transplant 2020;35:2015-9.

11 Rhee CM, Unruh M, Chen J, et al. Infrequent dialysis: a new paradigm for hemodialysis initiation. Semin Dial 2013;26:720-7.

12 Murea M, Moossavi S, Garneata L, et al. Narrative review of incremental hemodialysis. Kidney Int Rep 2020;5:135-48.

13 Kalantar-Zadeh K, Unruh M, Zager PG, et al. Twice-weekly and incremental hemodialysis treatment for initiation of kidney replacement therapy. Am J Kidney Dis 2014;64:181-6.

14 Obi Y, Kalantar-Zadeh K, Incremental K-ZK. Incremental and Onceto twice-weekly hemodialysis: from experience to evidence. Kidney Int Rep 2017;2:781-4.

15 Lee Y-J, Rhee CM, Kalantar-Zadeh K. Residual kidney function in twice-weekly hemodialysis: irreplaceable contribution to dialysis adequacy. Ann Transl Med 2018;6:317.
16 Gedney N, Kalantar-Zadeh K, Patient-Centeredness D. Dialysis Patient-Centeredness and precision medicine: focus on incremental home hemodialysis and preserving residual kidney function. Semin Nephrol 2018;38:426-32.

17 Lin Y-F, Huang J-W, Wu M-S, et al. Comparison of residual renal function in patients undergoing twice-weekly versus three-timesweekly haemodialysis. Nephrology 2009;14:59-64.

18 Fernández-Lucas M, Teruel-Briones JL, Gomis-Couto A, et al. Maintaining residual renal function in patients on haemodialysis: 5-year experience using a progressively increasing dialysis regimen. Nefrologia 2012;32:767-76.

19 Zhang M, Wang M, Li H, et al. Association of initial twice-weekly hemodialysis treatment with preservation of residual kidney function in ESRD patients. Am J Nephrol 2014;40:140-50.

20 Mathew A, Obi Y, Rhee CM, et al. Treatment frequency and mortality among incident hemodialysis patients in the United States comparing incremental with standard and more frequent dialysis. Kidney Int 2016;90:1071-9.

21 Bowline IG, Russell GB, Bagwell B, et al. Temporal trends in fluid management with incremental hemodialysis. Clin Nephrol. 2019;92:165-73.

22 Weir MR, Bakris GL, Bushinsky DA, et al. Patiromer in patients with kidney disease and hyperkalemia receiving RAAS inhibitors. $N$ Engl J Med 2015;372:211-21.

23 Bakris GL, Pitt B, Weir MR, et al. Effect of Patiromer on serum potassium level in patients with hyperkalemia and diabetic kidney disease: the AMETHYST-DN randomized clinical trial. JAMA 2015;314:151-61.

24 Pitt B, Bakris GL, Bushinsky DA, et al. Effect of patiromer on reducing serum potassium and preventing recurrent hyperkalaemia in patients with heart failure and chronic kidney disease on RAAS inhibitors. Eur J Heart Fail 2015;17:1057-65.

25 Kovesdy CP, Regidor DL, Mehrotra R, et al. Serum and dialysate potassium concentrations and survival in hemodialysis patients. Clin J Am Soc Nephrol 2007;2:999-1007.

26 Kovesdy CP, Anderson JE, Kalantar-Zadeh K. Association of serum bicarbonate levels with mortality in patients with non-dialysisdependent CKD. Nephrol Dial Transplant 2009;24:1232-7.

27 Wu DY, Shinaberger CS, Regidor DL, et al. Association between serum bicarbonate and death in hemodialysis patients: is it better to be acidotic or alkalotic? Clin J Am Soc Nephrol 2006;1:70-8.

28 Negi S, Koreeda D, Kobayashi S, et al. Renal replacement therapy for acute kidney injury. Renal Replacement Therapy 2016;2:31.

29 Vanholder R, Pletinck A, Schepers E, et al. Biochemical and clinical impact of organic uremic retention solutes: a comprehensive update. Toxins 2018;10:33.

30 Clark WR, Dehghani NL, Narsimhan V, et al. Uremic toxins and their relation to dialysis efficacy. Blood Purif 2019;48:299-314.

31 Watson PE, Watson ID, Batt RD. Total body water volumes for adult males and females estimated from simple anthropometric measurements. Am J Clin Nutr 1980;33:27-39.

32 Daugirdas JT. Estimating time-averaged serum urea nitrogen concentration during various urine collection periods: a prediction equation for Thrice Weekly and biweekly dialysis schedules. Semin Dial 2016;29:507-9.

33 Gotch FA. The current place of urea kinetic modelling with respect to different dialysis modalities. Nephrol Dial Transplant 1998;13(Suppl 6):10-14.

34 Evenepoel P, Bammens B, Verbeke K, et al. Superior dialytic clearance of beta(2)-microglobulin and p-cresol by high-flux hemodialysis as compared to peritoneal dialysis. Kidney Int 2006;70:794-9.

35 Garred LJ, Tang W, Barichello DL, et al. Equations for the calculation of the protein catabolic rate from predialysis and postdialysis urea concentrations and residual renal clearance in stable hemodialysis patients. Blood Purif 1997;15:157-68.

36 Hawthorne G, Richardson J, Osborne R. The assessment of quality of life (AQoL) instrument: a psychometric measure of health-related quality of life. Qual Life Res 1999;8:209-24.

37 Weisbord SD, Fried LF, Arnold RM, et al. Development of a symptom assessment instrument for chronic hemodialysis patients: the dialysis symptom index. J Pain Symptom Manage 2004;27:226-40.

38 Watnick S, Wang P-L, Demadura T, et al. Validation of 2 depression screening tools in dialysis patients. Am J Kidney Dis 2005;46:919-24.

39 Kroenke K, Spitzer RL, Williams JBW, et al. The patient health questionnaire somatic, anxiety, and depressive symptom scales: a systematic review. Gen Hosp Psychiatry 2010;32:345-59.

40 Spitzer RL, Kroenke K, Williams JBW, et al. A brief measure for assessing generalized anxiety disorder: the GAD-7. Arch Intern Med 2006;166:1092-7. 
41 Borson S, Scanlan J, Brush M, et al. The mini-cog: a cognitive 'vital signs' measure for dementia screening in multi-lingual elderly. Int $J$ Geriatr Psychiatry 2000;15:1021-7.

42 Daugirdas JT, Depner TA, Greene T, et al. Standard Kt/Vurea: a method of calculation that includes effects of fluid removal and residual kidney clearance. Kidney Int 2010;77:637-44.

43 Isakova T, Nickolas TL, Denburg M, et al. KDOQI US Commentary on the 2017 KDIGO Clinical Practice Guideline Update for the Diagnosis, Evaluation, Prevention, and Treatment of Chronic Kidney Disease-Mineral and Bone Disorder (CKD-MBD). Am J Kidney Dis 2017;70:737-51.

44 Fishbane S, Spinowitz B. Update on anemia in ESRD and earlier stages of CKD: core curriculum 2018. Am J Kidney Dis 2018;71:423-35.

45 Whitehead AL, Julious SA, Cooper CL, et al. Estimating the sample size for a pilot randomised trial to minimise the overall trial sample size for the external pilot and main trial for a continuous outcome variable. Stat Methods Med Res 2016;25:1057-73.

46 Williams JR. The Declaration of Helsinki and public health. Bull World Health Organ 2008;86:650-1.

47 International Committee of Medical Journal Editors. Recommendations for the conduct, reporting, editing, and publication of scholarly work in medical journals, 2019.

48 Newcombe RG. Two-sided confidence intervals for the single proportion: comparison of seven methods. Stat Med 1998;17:857-72.

49 Chin Al, Depner TA, Daugirdas JT. Assessing the adequacy of small solute clearance for various dialysis modalities, with inclusion of residual native kidney function. Semin Dial 2017;30:235-40.
50 John KA, Cogswell ME, Campbell NR, et al. Accuracy and usefulness of select methods for assessing complete collection of 24-hour urine: a systematic review. J Clin Hypertens 2016;18:456-67.

51 Shafi T, Michels WM, Levey AS, et al. Estimating residual kidney function in dialysis patients without urine collection. Kidney Int 2016;89:1099-110.

52 Wong J, Kaja Kamal RM, Vilar E, et al. Measuring residual renal function in hemodialysis patients without urine collection. Semin Dial 2017;30:39-49.

53 Chin Al, Sheth V, Kim J, et al. Estimating residual native kidney urea clearance in hemodialysis patients with and without 24-hour urine volume. Kidney Med 2019;1:376-82.

54 Kalantar-Zadeh K, Wightman A, Liao S. Ensuring Choice for People with Kidney Failure - Dialysis, Supportive Care, and Hope. N Engl J Med 2020;383:99-101.

55 Murea M. Precision medicine approach to dialysis including incremental and decremental dialysis regimens. Curr Opin Nephrol Hypertens 2021;30:85-92.

56 Hazara AM, Bhandari S. Can incremental haemodialysis reduce early mortality rates in patients starting maintenance haemodialysis? Curr Opin Nephrol Hypertens 2019;28:641-7.

57 Deira J, Suárez MA, López F, et al. IHDIP: a controlled randomized trial to assess the security and effectiveness of the incrementa hemodialysis in incident patients. BMC Nephrol 2019;20:8.

58 Kaja Kamal RM, Farrington K, Wellsted D, et al. Impact of incremental versus conventional initiation of haemodialysis on residual kidney function: study protocol for a multicentre feasibility randomised controlled trial. BMJ Open 2020;10:e035919.

59 Fernández Lucas M, Ruíz-Roso G, Merino JL, et al. Initiating renal replacement therapy through incremental haemodialysis: protocol for a randomized multicentre clinical trial. Trials 2020:21:206. 